$\begin{array}{cl}\begin{array}{c}\text { Revue } \\ \text { de /histoire } \\ \text { des religions }\end{array} & \text { Revue de l'histoire des religions } \\ & 1 \mid 2010 \\ & \text { Beauté du rite }\end{array}$

Séduction du rite et conversion par l'art, de Huysmans à Claudel

Ascendency of ritual and conversion through art, from Huysmans to Claudel

Catherine Mayaux

\title{
OpenEdition
}

1 Journals

Édition électronique

URL : http://journals.openedition.org/rhr/7565

DOI : $10.4000 /$ rhr.7565

ISSN : 2105-2573

Éditeur

Armand Colin

Édition imprimée

Date de publication : 1 mars 2010

Pagination : 93-108

ISBN : 978-2200-92612-0

ISSN : 0035-1423

Référence électronique

Catherine Mayaux, «Séduction du rite et conversion par l'art, de Huysmans à Claudel », Revue de

I'histoire des religions [En ligne], 1 | 2010, mis en ligne le 01 mars 2013, consulté le 19 avril 2019. URL: http://journals.openedition.org/rhr/7565; DOI : 10.4000/rhr.7565 


\section{Séduction du rite et conversion par l'art, de Huysmans à Claudel}

Les récits de conversion des années 1880 posent la question du lien entre l'art et la foi, la question du plaisir esthétique introduit au cœur de la prière : celui-ci rendrait le croyant coupable de céder à une séduction contraire à une véritable conversion. Mais Huysmans et Claudel montrent que l'art est nécessaire à la pratique religieuse et selon Claudel, qui propose une conception élargie à la vie de la liturgie, l'artiste créateur répond au Créateur.

\section{Ascendency of ritual and conversion through art, from Huysmans to Claudel}

Tales of conversion during the 1880s ask the question of the relationship between art and faith, of aesthetic pleasure within prayer: this would make the believer guilty to seduction as opposed to a true conversion. But Huysmans and Claudel show that art is necessary to religious practice and, according to Claudel, who proposes a conception of liturgy broadened to life, the creative artist responds to the Creator. 
Les récits de conversion des années 1880 , qu'ils soient romanesques ou autobiographiques, posent la question du lien entre l'art et la foi, la question de l'intrusion, au cœur de la prière et de la pratique religieuse, de la possible délectation esthétique : celle-ci rendrait le croyant coupable de céder à une séduction contraire à une véritable conversion ${ }^{1}$ qui suppose au contraire le dépouillement et l'abandon de toute forme matérielle et sensible, fût-elle de l'ordre de la beauté, au profit d'une vérité nue. Selon des modes d'expression très différents, le roman de Huysmans En Route (1895), le récit « Ma conversion» de Claudel (écrit en 1913, mais le premier choc de la conversion date de 1886), ainsi que divers textes de cet auteur sur la pratique liturgique, abordent explicitement le problème de la relation, tantôt heureuse, tantôt dévoyée, entre rite et art à la fin du XIX ${ }^{e}$ et dans la première moitié $\mathrm{du} \mathrm{Xx}^{\mathrm{e}}$ siècle. Le constat qu'ils font de l'évolution des pratiques sacramentaires en cette période charnière de fort courant antireligieux creuse en eux une inquiétude profonde quant à l'avenir d'une religion qui se couperait et de la tradition et de l'art qui ensemble ont fondé ses rites. Les enjeux sont d'autant plus importants que le bastion des fidèles, dont le nombre diminue, et les intellectuels et artistes croyants se trouvent pris en tenaille entre les tenants d'un positivisme qui considèrent la religion comme vouée à la disparition - à l'instar d'une espèce inadaptable selon le modèle darwinien -, et l'Église romaine qui tend à accentuer le divorce d'avec le monde moderne par ses encycliques et la multiplication des gestes de dévotion de toutes sortes. La question de la beauté du rite est donc prégnante dans leur réflexion d'artistes convertis et Claudel, sans doute mieux que Huysmans, mais avec une efficacité qu'on jugera peut-être limitée, en justifie le maintien ou le recours aux plans à la fois spirituel, esthétique et intellectuel.

1. Les deux termes disent par leur étymologie des orientations contraires : séduction, du verbe latin seducere, « emmener à part, hors du chemin »; conversion, de convertere, « tourner vers ». 


\section{« La VRaie preuve du Catholicisme »}

Entrant un soir de novembre dans l'église Saint-Sulpice, au moment où se célèbre l'octave des morts, Durtal, le héros d'En Route de Huysmans ${ }^{2}$, est saisi par la beauté du psaume chanté De profundis ad te clamavi, dont les « gerbes de voix », les «timbres pointus », les basses «comme souterraines» (p. 54) prennent possession du volume de l'église comme de la totalité de son âme et expriment, jusqu'à la douleur d'une déchirure intérieure, «l'âme désincarnée, jetée nue, en pleurs, devant son Dieu » (p. 55). Toute la réflexion du personnage le conduit à une vénération inextricablement conjointe du Sauveur et d'une religion fondée en art, une religion dont l'art «allaité par l'Église » dès l'Antiquité et le Moyen Âge était «la vraie preuve du Catholicisme » (p. 58). Le lexique psychique autant que médical employé pour décrire la soumission de Durtal à la loi religieuse et à Dieu, le pathétisme appuyé et anthropomorphisé de la description des chants, les images profuses suggérées par ces mêmes chants, même s'ils concèdent au goût à la fois symboliste et fin de siècle mâtiné du naturalisme de l'époque, naturalisme dont Huysmans avait d'abord épousé les principes ${ }^{3}$, rendent suspecte l'inquiétante emprise nerveuse et psychologique plus que spirituelle que cette excessive beauté exerce sur l'esprit de l'impétrant, soumis à l'envoûtement de ses sens ${ }^{4}$. La proclamation de foi conjointe en l'Église et en l'art, l'une nourrissant l'autre et réciproquement, fait la distinction entre des œuvres créées "par l'effluence mystique, par l'émanation même du Moyen Âge » et les créations modernes où l'on sent, dit Durtal, «l'artiste tapi sous son œuvre», point sur lequel nous reviendrons. ${ }^{5}$. Mais elle procède d'une radicale identification, sinon confusion, autant psychique que spirituelle, entre art et rite, la beauté du rite provoquant ou accroissant la foi du fait de

2. J.-K. Huysmans, En Route, éd. Dominique Millet, Paris, Gallimard, « Folio classiques », 1996. Les références de pages entre parenthèses renvoient à cette édition.

3. Voir « J.-K. Huysmans », in Léon Bloy, Les Funérailles du naturalisme, édition présentée, établie et annotée par Pierre Glaudes, Paris, Les Belles Lettres, 2001, p. 47-77.

4. «Cette requête sublime finissant dans les sanglots [...] tordit les nerfs de Durtal, lui tressailla le cour $[. .]$.$» p. 56; «Il finissait par être pris aux moelles,$ suffoqué par de nerveuses larmes [...] », p. 91 et passim.

5. Voir infra note 32. 
l'intensité de l'émotion esthétique, de l'effusion et de la dilatation intérieure due à la jouissance de la beauté, et non véritablement à la méditation du croyant ou à son adhésion à un dogme. Le récit de la conversion de Durtal est placé sous le signe de cette association troublante, du fait de son excès, entre rite et art : l'art apparaît comme plus efficace pour « remuer », « capter» et « asservir » son âme que le cheminement intellectuel et spirituel qu'il avait entamé, ce que l'abbé Gèvresin ne se fait pas faute de remarquer au chapitre V :

L'art a été le principal véhicule dont le Sauveur s'est servi pour vous faire absorber la Foi. Il vous a pris par votre côté faible... ou fort, si vous aimez mieux. Il vous a imprégné de chefs-d'œuvre mystiques; il vous a persuadé et converti, moins par la voie de la raison que par la voie des sens; et dame, ce sont là des conditions très spéciales dont il importe de tenir compte (p. 149).

Mais le constat était déjà le même dans le monologue intérieur de Durtal qui retrace l'itinéraire qui l'a conduit à «en arriver là » (p. 75) :

[...] par le son extérieur des mots, sans l'aide du recueillement, sans l'appui même de la réflexion, l'Église agit./Et c'est là le miracle de sa liturgie $[\ldots]$ (p. 72).

[...] Le jour où, par curiosité, pour tuer le temps, il était entré dans une église et, après tant d'années d'oubli, y avait écouté les Vêpres des morts tomber lourdement, une à une, tandis que les chantres alternaient et jetaient, l'un après l'autre, comme des fossoyeurs, des pelletées de versets, il avait eu l'âme remuée jusque dans ses combles. Les soirs où il avait entendu les admirables chants de l'octave des trépassés, à Saint-Sulpice, il s'était senti pour jamais capté; mais ce qui l'avait pressuré, ce qui l'avait asservi mieux encore, c'étaient les cérémonies, les chants de la semaine sainte (p. 85).

L'approche est plus discrète et mesurée dans le récit que fait Claudel de sa conversion (et l'analyse ultérieure qu'il proposera du rôle de l'art dans la liturgie et l'Église orientera autrement sa théorie); il n'en témoigne pas moins dans les interstices du texte de l'attrait exercé par la dimension esthétique du rituel sur l'âme disponible de ce « malheureux enfant qui, le 25 décembre 1886, se rendit à Notre-Dame de Paris pour y suivre les offices de Noël » :

Je commençais alors à écrire et il me semblait que dans les cérémonies catholiques, considérées avec un dilettantisme supérieur, je trouverais un excitant approprié et la matière de quelques exercices décadents. C'est dans ces dispositions que, coudoyé et bousculé par la foule, j'assistai avec un plaisir médiocre, à la grand'messe. Puis, 
n'ayant rien de mieux à faire, je revins aux vêpres. Les enfants de la maîtrise en robes blanches et les élèves du petit séminaire de SaintNicolas du Chardonnet qui les assistaient, étaient en train de chanter ce que je sus plus tard être le Magnificat. J'étais moi-même debout dans la foule, près du second pilier à l'entrée du chœur à droite du côté de la sacristie. Et c'est alors que se produisit l'événement qui domine toute ma vie. En un instant je fus touché et je crus. [...] Les larmes et les sanglots étaient venus et le chant si tendre de l'Adeste ajoutait encore à mon émotion ${ }^{6}$.

Le souvenir visuel et auditif en est suffisamment vif pour que Claudel y revienne plus de quarante ans après, en 1938, dans une lettre à Henri Reynaud qui sollicite un « avant-dire » pour le volume qu'il vient de consacrer à la Manécanterie des Petits Chanteurs à la Croix de bois, marquant une fois de plus la part de cette dimension sensorielle dans l'émotion qui l'a alors saisi :

Comment pourrais-je vous le refuser, alors que ce sont les frères de vos petits chanteurs revêtus de la même aube et du même amict, qui, un certain soir de décembre, à Notre-Dame de Paris, ont purifié mon âme des ténèbres matérialistes? Puissance merveilleuse de la voix, de l'âme directement qui atteint, qui imprègne, qui s'unit l'autre âme et qui l'entraîne avec elle, avec la connivence irrésistible de l'oreille, dans un même acte d'amour, quand c'est l'amour qui va à la rencontre de la foi!

Claudel évoque encore plus loin le rôle joué par « ces êtres innocents qui énonçaient l'un après l'autre [...] les divines affirmations d'un Magnificat ${ }^{7}$ » et son propos rappelle au lecteur les «voix d'enfants tendues jusqu'à éclater, ces voix claires et acérées [qui] mettaient dans la ténèbre du chant des blancheurs d'aube [...] » qui séduisent Durtal dans En Route (p. 55-56).

\section{« L’ART ET LA POÉSIE AUSSI SONT DES CHOSES DIVINES »}

L'attrait esthétique chez Claudel n'est toutefois pas une donnée préalable à cette conversion, ni ne relève d'une aspiration à

6. «Ma conversion », Contacts et circonstances, Euvres en prose, édition établie et annotée par Jacques Petit et Charles Galpérine, Paris, Gallimard, « Bibliothèque de la Pléiade », 1965, p. 1010. Nous soulignons, mais les italiques de « je crus » sont le fait de Claudel.

7. «Le Chant religieux», lettre du 14 novembre 1938 adressée à Henri Reynaud, Euvres complètes, Paris, Gallimard, 1959, t. XVI, p. 204-208. 
laquelle il céderait comme Huysmans, "l'homme le plus idolâtre d'art que j'ai rencontré dans ma vie » selon le témoignage de Léon Bloy dans les années 1890-1891. S'il dit envisager d'écrire, sa vocation poétique profonde se déclenche et se consolide au moment de cette conversion, progressant en même temps que sa vocation de croyant; le plaisir d'esthète dont il témoigne avant sa conversion est emprunté à l'air du temps, superficiel et surfait (par un « dilettantisme supérieur »; « la matière de quelques exercices décadents »), et reste en deçà de la dévotion à l'art de Durtal. S'opposent sur ce point En Route: «Enfin Durtal avait été ramené à la religion par l'art. Plus que son dégoût de la vie même, l'art avait été l'irrésistible aimant qui l'avait attiré vers Dieu » (p. 85), et le propos de Claudel : «L'étude de la religion était devenue mon intérêt dominant. Chose curieuse! L'éveil de l'âme et celui des facultés poétiques se faisait $[s i c]^{8}$ chez moi en même temps » (" Ma conversion » p. 1012) et plus loin : «Peu à peu, lentement et péniblement, se faisait jour dans mon cœur cette idée que l'art et la poésie aussi sont des choses divines $[\ldots] »($ p. 1013).

Marqués qu'ils sont par la lecture régénérante de L'Année liturgique $^{9}$ de Dom Guéranger, l'abbé de Solesmes, qui veut réintroduire la dimension esthétique dans la liturgie ${ }^{10}$, Claudel et Huysmans montrent tous deux leur sensibilité à la dynamique proactive du rite dans sa dimension esthétique et à l'effet qu'il provoque, même si chez Claudel ce sentiment va très vite s'élever à un niveau intellectuel et spirituel. Tous deux contextualisent fortement ces événements, ce n'est pas indifférent, dans le dernier quart du XIX $X^{e}$ siècle, celui de l'âge mûr pour Huysmans, de la jeunesse pour Claudel, époque qui connaît une double crise esthétique et religieuse, la conjonction des deux se révélant catastrophique pour l'Église. L'anticléricalisme bat son plein, en même temps que la déchristianisation progresse à grands pas dans les dernières décennies du siècle, et Huysmans comme Claudel, et comme bien d'autres en

8. Le texte de Gallimard met bien le verbe au singulier; si ce singulier a été voulu par Claudel au lieu du pluriel attendu, il est significatif.

9. Dont l'édition débute en 1841 et connaitra un grand succès, une fois achevée, à partir de 1875 .

10. Voir à ce sujet la préface de D. Millet à En Route (op. cit.) et du même auteur, « Poëme biblique et poétique sacramentelle chez Paul Claudel », La Prose transfigurée. Études en hommage à Paul Claudel, PUPS, 2005, p. 301-302; ainsi que Bertrand Marchal, La Religion de Mallarmé, José Corti, 1988, p. 31. 
cette fin de siècle (Goncourt, Zola, Flaubert...) raillent ce que sont devenues les pratiques religieuses, au point pour Claudel de répugner à l'idée de se mêler aux fidèles : " la religion catholique me semblait toujours le même trésor d'anecdotes absurdes, ses prêtres et les fidèles m'inspiraient la même aversion qui allait jusqu'à la haine et jusqu'au dégoût» (p. 1010). Dans «Le goût du fade» comme dans la «Lettre à Alexandre Cingria sur les causes de la décadence dans l'art sacré », il met en cause l'inaptitude à l'art des prêtres $^{11}$, « la crise d'une imagination mal nourrie ${ }^{12}$ », la tendance à la facilité qui dévoie l'art sacré vers un souci de plaire ${ }^{13}$, et le fait, absurde à ses yeux, de s'adresser pour embellir l'Église et la pratique liturgique, non pas aux meilleurs artistes dont regorge le siècle (Rude, Carpeaux, Rodin, Bourdelle, Maillol, Despiau), mais « à des marbriers de cimetières et de lavabos, fournisseurs de simulacres désossés ${ }^{14} »$. Mêmes lamentations chez Huysmans : interrogé par le journal Le Sillon le 16 janvier 1899 dans le cadre d'une « enquête sur la renaissance idéaliste » sur la capacité du public catholique à s'élever « au point de vue littéraire et artistique », il répondit ceci :

[...] le public catholique se tranche en deux parts très inégales. Une, minuscule, celle des gens pieux, intelligents et lettrés, épris de mystique et d'art, considérés par les autres comme des toqués et des êtres dangereux, et une autre, énorme, composée de toute la tourbe des catholiques ignares et bégueules, très décidés à empêcher, par tous les moyens possibles, toute tentative de littérature et tout essai d'art. / L'idéal de la rue Saint-Sulpice leur suffit parfaitement et ils n'en veulent aucun autre. Ce sont les médiocres dont parlait Hello, les Pharisiens, les inéducables Pharisiens ${ }^{15}$.

De même son personnage raille dévots et cagots qu'il voit dans les églises (p. 102-103) et déplore la laideur sacrilège des basiliques modernes, « inertes », « sourdes aux prières qui se brisaient contre l'indifférence glacée de leurs murs » (p. 93), et plus encore l'igno-

11. «Il faut s'en prendre aussi au clergé qui pendant trop longtemps s'est confiné vis-à-vis du siècle dans une attitude apeurée et défensive et a cru que l'art ne pouvait se séparer du péché qu'en se séparant de la vie» dans «Le goût du fade », Positions et Propositions, Euvres en prose, op. cit., p. 116.

12. «Lettre à Alexandre Cingria sur les causes de la décadence dans l'art sacré », Positions et Propositions, loc. cit., p. 119.

13. « Le goût du fade», loc. cit., p. 117.

14. Ibidem.

15. «Enquête sur la renaissance idéaliste », Le Sillon, 16 janvier 1899, reproduit par D. Millet dans En route, édition citée, p. 558. 
minie de la musique et des chants quand ils ne sont pas plain-chant ou psaumes :

À Saint-Étienne-du-Mont, c'était pis encore; la coque de l'église était charmante mais la maîtrise était une succursale de la maison Sanfourche; on se serait cru dans un chenil où grognait une meute variée de bêtes malades; quant aux autres sanctuaires de la rive gauche, ils étaient nuls; l'on y supprimait autant que possible le plain-chant, et partout l'on y embrenait avec des fredons libertins la pauvreté des voix (p. 96).

Plus loin il stigmatise les églises où « presque toujours on habille le Tantum ergo de flons-flons imbéciles, de mélodies pour fanfare militaire et pour banquet ", s'en prenant au clergé qui accepte de tels sacrilèges :

[...] il fallait réellement que les curés eussent perdu, non pas le sens de l'art - puisqu'ils ne l'ont jamais eu -, mais le sens le plus élémentaire de la liturgie, pour accepter de semblables hérésies, pour supporter de pareils attentats dans leurs églises! (p. 174).

À cet état de désesthétisation du rite auquel est parvenue selon Huysmans et Claudel la liturgie en cette fin de siècle s'ajoute l'atmosphère de « ces tristes années quatre-vingts, l'époque du plein épanouissement de la littérature naturaliste » comme l'explique Claudel dans « Ma conversion » :

Jamais le joug de la matière ne parut mieux affermi. Tout ce qui avait un nom dans l'art, dans la science et dans la littérature était irréligieux. Tous les (soi-disant) grands hommes de ce siècle finissant s'étaient distingués par leur hostilité à l’Église. Renan régnait (p. 1009).

La question de la beauté du rite est donc à leurs yeux cruciale (le mot ne convient-il pas?) et déterminante pour l'avenir de l'Église et du monde croyant. Pour Huysmans « la Grâce se mêl[e] aux éloquentes splendeurs des liturgies » (En Route, p. 86) et pour Claudel, « l'art fait partie du rituel » :

Il [1'art] a pour but non pas de nous évangéliser et de nous instruire, d'exciter nos sentiments, ou directement de glorifier Dieu, mais de constituer autour de la Croix, autour de la Panagia ou du Pantocrator, un ensemble de présences officielles, une cour, un chœur, un clergé permanent sur les murailles, un mobilier pictural, la garde plate de l'iconoclaste, la délégation des Anges et des Saints à laquelle les jours de fête vient s'adjoindre la foule terrestre. L'Église est une basilique, c'està-dire la résidence d'un Souverain au milieu de sa cour ${ }^{16}$.

16. « Note sur l'art chrétien », loc. cit., p. 121. 


\section{IN HYMNIS ET CANTICIS}

Si tous les arts doivent participer d'après eux à la beauté de la liturgie et construire cette basilique à la gloire de Dieu : architecture, sculpture, peinture, musique, poésie (auxquels peuvent s'adjoindre toutes les formes de l'artisanat : mobilier d'église, vêtements sacerdotaux et d'apparat religieux, objets du culte et pièces ornementales...), ils récusent catégoriquement le style sulpicien qui sévit à leur époque ${ }^{17}$, de même que les musiques de leur modernité, « mystique égrillarde, ces fonts à l'eau de bidet qu'inventa Gounod » (En Route, p. 66) ou « qui donne des signes évidents d'anémie et de décrépitude $\|^{18}$; ils en appellent à l'art médiéval, remontant aux hautes époques du christianisme, à savoir sa naissance et son émergence à travers la constitution du canon biblique et les textes fondateurs des Pères de l'Église; et son renouveau au cours des $\mathrm{XII}^{\mathrm{e}}$ et $\mathrm{XIII}^{\mathrm{e}}$ siècles, grands siècles d'une théologie qui s'enrichit de la philosophie ainsi que du renouveau de la vie ecclésiale avec l'apparition de plusieurs ordres monachiques amenés à jouer un rôle éminent dans la vie religieuse ${ }^{19}$. Aussi saint Paul est-il convoqué par Claudel : « où nos forces trouveraientelles meilleure expression que dans ces hymnes et cantiques spirituels que nous recommande saint Paul?»; aussi en appelle-t-il au fil des années à l'antienne et à l'hymne, à l'ancienne liturgie gallicane :

Que de trésors contient cette musico-littéraire [prose] franco-latine aujourd'hui si misérablement oubliée et abandonnée! Là est une des sources et des ressources les plus profondes de notre poésie, la plus intimement associée à notre génie national. Qui rend justice aujourd'hui à ce magnifique poète qu'est Adam de Saint-Victor? (Et la même abbaye produisit plus tard le grand Santeul) ${ }^{20}$.

Ce même nom de Saint-Victor et d'autres réapparaissent sous sa plume lorsqu'il rédige le chapitre VII de son commentaire de l'Apo-

17. « Cette bondieusarderie qui s'épand le long de la rue de Saint-Sulpice », En Route, p. 107.

18. «Le Chant religieux », loc. cit., p. 207.

19. Voir à ce sujet, André Haquin, "Parcours à la découverte de la ritualité chrétienne », Rite et littérature, Cahiers électroniques de l'imaginaire $\mathrm{n}^{\circ} 3$, Myriam Watthee-Delmotte et David Martens éd., www.e-montaigne.com, automne 2005 , p. $5-15$.

20. " Le Chant religieux », loc. cit., p. 206. 
calypse de saint Jean: "Les hymnes d'Ambroise, les psaumes de David, les cantilènes de Grégoire, les séquences de Nottker [sic], les proses du moine de Saint-Victor, mouillent mes yeux et pénètrent notre moelle ${ }^{21}$. 》 Claudel décline là un pan de l'histoire de l'hymnodie latine et de la naissance de la toute première poésie de langue française : Ambroise (337-397), évêque de Milan au $\mathrm{IV}^{\mathrm{e}}$ siècle, composa des hymnes à partir de la tradition poétique latine afin d'encourager la pratique des fidèles, hymnes qui devinrent si populaires que la postérité a tendu à le considérer comme le fondateur de l'hymnologie latine (il serait l'auteur du Te Deum); on a tardivement attribué à Grégoire le Grand (540-604), pape romain en 590, la codification du chant rituel ou chant grégorien; Netker Balbulus («le Bègue», appellation due aux formes répétées de sa musique, non à un défaut d'élocution), moine de Saint-Gall en Suisse, écrivit des hymnes à saint Étienne et une quarantaine de séquences; on sait enfin combien l'école de Saint-Victor signa une période féconde du développement de l'art et de la pensée religieuse au XII ${ }^{\mathrm{e}}$ siècle, marquée autant par les études théologiques et exégétiques, que par la créativité en poèmes liturgiques dont Adam fut un remarquable représentant ${ }^{22}$. Huysmans remonte à travers les pensées de son personnage au « chant liturgique créé presque toujours anonymement au fond des cloîtres, [...] source extraterrestre, sans filon de péchés, sans trace d'art. [...] c'était aussi l'idiome de l'Église, l'Évangile musical accessible comme l'Évangile même, aux plus raffinés et aux plus humbles » (p. 58); ou au Dies irae anonyme, "simplement form[é] par les alluvions douloureuses des temps » et qui « semblait être tout d'abord tombé, ainsi qu'une semence de désolation, dans les âmes éperdues du $\mathrm{XI}^{\mathrm{e}}$ siècle » (p. 64-65). Tous deux considèrent que la pratique religieuse et la prière doivent s'accomplir par cet art sacré, et Claudel appelle de ses vœux un retour à cette haute époque liturgique, demandant : «Est-il possible de rêver que les temps futurs assisteront dans nos

21. L'Apocalypse de saint Jean, dans Le Poëte et la Bible, I, 1910-1946, édition établie, présentée et annotée par Michel Malicet, avec la collaboration de Dominique Millet-Gérard et Xavier Tilliette, Paris, Gallimard, 1998, p. 1159.

22. Voir "L'École de Saint-Victor", Dictionnaire critique de théologie, Jean-Yves Lacoste (dir.), PUF, 1998, p. 1041-1043. Voir aussi Isabelle RenaudChamska, "Poétique de La Liturgie des Heures, Notes brèves sur le Bréviaire », Poésie et Liturgie, $\mathrm{xIx}^{\mathrm{e}}-\mathrm{xx}^{\mathrm{e}}$ siècles, Martine Bercot et Catherine Mayaux (éd.), Peter Lang, 2006, p. 177-197. 
églises à la renaissance de la prose ${ }^{23}$, ancienne et moderne, qui remplacerait avantageusement tant de fades motets $^{24}$ ?» On sait que l'histoire de l'Église n'accomplira pas son rêve et qu'il témoignera de sa déception au fil du temps dans son Journal; en avril 1953 il note : «La nouvelle liturgie laisse le Samedi-Saint complètement vide. [...] La grande solennité avec laquelle nous étaient apportés les sacrements du Feu et de l'Eau est bousculée. Pâques est dégradée comme l'est aujourd'hui Noël par ces tristes messes de minuit »; et le jour de l'Assomption : « N[ous] avons subi une dégradation. [...] La messe latine n'est pas faite pour cela et en tant qu'œuvre d'art elle subit un affreux dommage. Sans parler de cet odieux mélange de français. Cela ressemble beaucoup à un "culte" protestant. Trouble et tristesse »; au point que le 29 janvier 1955, quelques jours avant sa mort (survenue le 23 février - c'est dire l'inquiétude que constituait pour le poète croyant l'évolution de la liturgie), il publie un article dans le Figaro Littéraire intitulé $L a$ Messe à l'envers dans lequel il proteste virulemment contre les nouvelles modalités de la liturgie qui « dépouille le peuple chrétien de sa dignité et de son droit», regrettant encore que « le déplorable usage actuel a[it] complètement bouleversé l'antique cérémonial au plus grand trouble des fidèles ${ }^{25} »$. À travers la critique de la métamorphose de l'autel devenu un «vague tréteau recouvert d'une nappe qui rappelle douloureusement l'établi calviniste » pointe aussi le rejet de la Réforme et du culte protestant dont Claudel a toujours dit le mal qu'il en pensait ${ }^{26}$ et le désir farouche de se distinguer de celui-ci en maintenant l'ancienne liturgie ou celle de l'époque dite baroque riche en ornements et en chants. Dans la deuxième Journée

23. On peut imaginer que Claudel utilise ici le terme «prose » en son sens médiéval synonyme de "séquence», forme psalmique composée de versets qui « suit » 1'Alleluia à la messe; voir à ce sujet Dominique Millet-Gérard, « Claudel hymnode et séquentiaire, le secret de l'iambe », Paul Claudel 18, Minard, Revue des Lettres modernes, 2003, p. 120.

24. " Le Chant religieux », loc. cit., p. 206.

25. Paul Claudel, Journal, t. II, 1923-1955, texte établi et annoté par François Varillon et Jacques Petit, Paris, Gallimard, « Bibliothèque de la Pléiade », 1969, respectivement p. 832, 842 et 885 . «La messe à l'envers », Supplément aux CEuvres complètes, Lausanne, L’Âge d'Homme, 1990, t. I, p. 294-295.

26. Voir les propos de saint Boniface dans Le Soulier de Satin, III, 1, Théâtre, textes et notices établis par Jacques Madaule et Jacques Petit, Paris, Gallimard, «Bibliothèque de la Pléiade », 1965, p. 785-786; voir aussi notre article, « Claudel poète de la guerre », Claudel politique, Pascal Lecroart éd., Lons-Le-Saunier, Aréopage, 2009, p. 103-116. 
du Soulier de Satin, le Vice-Roi critique les « tristes réformateurs » précisément parce qu'ils réduisent leur pratique religieuse «à cette transaction personnelle et clandestine dans un étroit cabinet,/ Blasphémant que les œuvres ne servent pas, celles de Dieu sans doute pas plus que celles de $1^{\prime}$ homme ${ }^{27} »$, et condamne donc clairement - au nom de Claudel - la doctrine de la sola fides. La critique du calvinisme s'accompagne d'ailleurs de manière sous-jacente d'une pointe d'anti-germanisme - fréquente sous la plume de Claudel - qui explique l'association qu'il fait ici et là (voir citations supra) entre la beauté des anciennes hymnes et l'idée d'un génie national et gallican. La « Lettre à Alexandre Cingria sur les causes de la décadence de l'art sacré » professe clairement son attachement à l'esthétique baroque, à «l'art postérieur au concile de Trente», donc à l'art de la Contre-Réforme :

[...] l'art postérieur au concile de Trente et connu généralement sous le nom absurde d'art baroque, pour qui d'ailleurs j'éprouve, comme vous-même, vous le savez, la plus vive admiration, semble avoir pris pour objet [...] de montrer avec bruit, avec faste, avec éloquence, et souvent avec le pathétique le plus émouvant, cet espace vacant comme un médaillon et dont l'accès est interdit à nos sens pompeusement congédiés. Et ce sont des saints qui par le visage et l'attitude nous indiquent l'ineffable et l'invisible, et toute la foison désordonnée de l'ornement, et des anges qui dans le tourbillon d'ailes soutiennent un tableau indistinct et dissimulé par le culte, et des statues comme remuées par un grand souffle qui vient d'ailleurs ${ }^{28}$.

L'enjeu des questions esthétiques et de l'art liturgique est donc de première importance aux yeux de Claudel, et nul doute qu'il n'eût vigoureusement condamné la rénovation de la liturgie réalisée par les réformes de Vatican II à partir de 1963. La référence que fait Claudel à la vieille hymnodie latine ou franque dit son attachement à la pratique la plus ancienne de la liturgie qui correspond en même temps à la naissance de la poésie française, émanation du christianisme même : celle-ci est à ce titre justifiée par celle-là, et est par essence empreinte d'un souci de Dieu, liée à la volonté de Dieu.

27. Le Soulier de Satin, II, 5, op. cit., p. 748-749.

28. «Lettre à Alexandre Cingria... », loc. cit., p. 119. 


\section{« RÉPondre Au Créateur PAR Une CRÉATION »}

Claudel s'est attaché lui-même à inscrire sa poésie comme sa poétique dans la continuation de l'ancienne poésie religieuse. Dans «Claudel hymnode et séquentiaire, le secret de l'ïambe », Dominique Millet-Gérard expose les hésitations du poète quant à la forme qu'il recherche pour les poèmes qui formeront en 1914 la Corona benignitatis anni Dei : hésitant entre le modèle de la séquence et celui des hymnes, il choisirait de combiner forme strophique et vers psalmique ${ }^{29}$. Cette étude met aussi en valeur l'accord profond que dégage Claudel entre la rythmique de la poésie liturgique du Moyen Âge dans son héritage latin et la théorie de l'ïambe essentielle à la poétique claudélienne développée dans son Art poétique et dans « Réflexions et Propositions sur le vers français ». Elle rappelle aussi le lien entre cette théorie de l'iambe et le mouvement même de la vie perçu et conçu par Claudel :

Mais le vers dont l'emploi partout a prévalu est le vers ïambique universellement employé dans le théâtre antique et dont l'élément unique (couple d'une brève et d'une longue) est la traduction la plus simple de cette pulsation qui ne cesse de compter le temps dans notre poitrine.

L'expression sonore se déploie dans le temps et par conséquent est soumise au contrôle d'un instrument de mesure, d'un compteur. Cet instrument est le métronome intérieur que nous portons dans notre poitrine. Le coup de notre pompe à vie $[\ldots]$ /L'iambe fondamental, un temps faible, un temps fort ${ }^{30}$.

Cette présence conjointe dans la pensée claudélienne d'une conception du rythme poétique fondamental, de la théorie de l'iambe, d'une tension pour pratiquer la poésie dans la mémoire de l'ancienne hymnodie, et du référent rythmique vital propre à tout homme (rythme cardiaque et circulation sanguine) qui fonde cette poétique donne une exceptionnelle cohérence tout à la fois à la pensée religieuse de Claudel et à sa pensée de la poésie : elles sont indissoluble-

29. «[...] comme si Claudel superposait le modèle d'Adam de Saint-Victor, dont la forme est [...] strophique, le vers court (le plus souvent l'octosyllabe hérité de saint Ambroise, avec des chutes en heptasyllabes), et la rime presque systématique, et celui de Notker, qu'il lit chez Dom Guéranger sous forme de vers long assonancé, alors que les éditeurs modernes ont tendance à le fragmenter en unités rythmiques plus brèves $[\ldots]$ » « Claudel hymnode et séquentiaire, le secret de l'ïambe », op. cit., p. 121 ; voir aussi p. 125.

30. "Réflexions et propositions sur le vers français », Euvres en prose, op. cit., respectivement p. 6-7 et p. 5 . 
ment liées. Sa conception de la poésie revêt de ce fait une dimension universaliste qui autorise celle-ci à prendre place, au moins pour la partie de ses poèmes religieux, aux côtés des chants, anonymes ou non, de la liturgie catholique. Un article suggestif de Nathalie Nabert « Prière et poésie chez quelques poètes $\mathrm{du} \mathrm{Xx}^{\mathrm{e}}$ siècle " met ainsi en lumière l'étroite parenté entre certains poèmes de Claudel et certains psaumes, fondée notamment sur la réactualisation d'une hérédité qui assume passé et futur, mettant à distance la dimension personnelle de la parole ${ }^{31}$. La question de l'auteur, celle du sujet lyrique et de la singularité sont rendues secondaires tout à la fois par cette théorie d'un rythme fondamental propre à tout être vivant, et par l'englobement de toute création dans le mouvement de la Création, par l'inscription de la parole poétique dans la genèse d'une parole religieuse (im)mémorielle. Dans sa « Note sur l'art chrétien », Claudel s'interroge à propos de la « vierge de bazar issue d'un moule à saucisses » à laquelle la Carmélite et la Petite Sœur des Pauvres adressent leurs « confidences ravissantes » et sur ce qui pourrait justifier un art aussi déplorable offert à la dévotion :

Faut-il penser que ces statues impersonnelles, si elles ne rassasient pas la dévotion, du moins elles ne lui font pas d'obstacle, elles n'interposent pas entre Dieu et nous la gênante personnalité de l'artiste ${ }^{32}$ ? Faut-il croire [...] que par leur laideur même, par leur ignominie sans nom, elles sont plus propres à nous parler de la Beauté incommunicable? Là où l'homme, fût-ce un homme de génie, tient plus de place, il y a moins de place pour $\operatorname{Dieu}^{33}$.

Claudel récuse évidemment cette idée, lui qui a dévolu son âme à Dieu et sa vie à la création poétique. Aussi poursuit-il, et il nous paraît important de le citer longuement :

Ce sont là des idées que ses sentiments professionnels rendent bien difficile à un artiste d'accepter. Il s'indigne et se désole de voir toute la Création aujourd'hui déshéritée de cette parole de reconnaissance et d'amour qu'elle doit à son Créateur. [...] il sait que la Beauté ne peut être la récompense d'une inspiration hasardeuse, mais l'effet - ou

31. L'auteur rapproche notamment le poème "Ténèbres" de la section «Images et signets entre les feuilles» de la Corona et le psaume 17. Nathalie Nabert, «Prière et poésie chez quelques poètes du $\mathrm{Xx}^{\mathrm{e}}$ siècle », Revue de l'Institut catholique de Paris, Transversalités, $\mathrm{n}^{\circ}$ 61, janvier-mars 1997, p. 53-65, voir en particulier p. 59.

32. Nous retrouvons ici la réflexion de Durtal envisagée au début de notre travail.

33. « Note sur l'art chrétien », loc. cit., p. 128. 
le présage? - d'un mouvement général de l'horizon, d'une poussée de l'Univers, de la reprise jusque dans ses fondations de l'œuvre des Six Jours. [...] L'élan, l'effort honnête d'un artiste, qui, n'importe comment et de son mieux, avec les moyens qu'il a à sa disposition, essaye non pas lui-même d'apparaître, mais de répondre, de répondre à la parole par une parole, à la question par un acte, et au Créateur par une création, cet effort ne peut pas rester isolé et produit, à l'insu ou non de son auteur, autour de lui, toutes sortes de conséquences et de propositions constructives ${ }^{34}$.

L'écriture poétique, comme l'art, est « parole de reconnaissance et d'amour » : « S. François en flammes fait sortir Giotto de son baquet de plâtre et Giotto derrière lui tire tout l'art italien ${ }^{35} »$; l'art comme la poésie sont prière et répons : «Leur prière, c'est l'opération de leur art $^{36}$. » Paul Claudel répond les psaumes est le titre choisi par le poète pour sa traduction des psaumes publiée pour la première fois en 1948 chez Ides et Calendes ${ }^{37}$. D'où l'importance qu'accorde Claudel dans la pratique liturgique au fait que le peuple chrétien dise la messe avec le prêtre (et non le prêtre pour l'assistance ni face à elle) qui « se retourne de temps à autre pour s'assurer de sa présence, de sa participation et de sa coopération ${ }^{38} »$, de l'importance qu'il accorde à la vérité de l'échange entre les deux groupes d'officiants, prêtre et peuple, à travers les Dominus vobiscum et Orate, fratres :

Le Curé (dans cette église de Paris que je sais), après qu'il a chanté le Credo, quand il dit: Dominus vobiscum,

Se retourne vers l'assistance [...] moi?»

« Le Seigneur est avec vous, mes frères! Mes frères, êtes-vous avec

tonne l'officiant dans La Messe là-bas ${ }^{39}$ attendant le répons et les chants des fidèles. Quant aux conséquences de toutes sortes et aux « propositions constructives » évoquées par Claudel, il suffit de

34. Ibid., p. 128, 129 et 130.

35. Ibid., p. 130.

36. Ibid., p. 131.

37. Voir à ce sujet l'article d'Aurélien Gleize, «À propos des traductions des psaumes par Paul Claudel », Poésie et liturgie, XIX'-XXe siècles, op. cit., p. 117-135.

38. Voir La Messe à l'envers, op. cit.

39. Paul Claudel, La Messe là-bas, Euvre poétique, Textes établis et annotés par Jacques Petit, «Bibliothèque de la Pléiade », Paris, Gallimard, 1967, p. 502. Voir « Modèles liturgiques et enjeux poétiques dans "La Messe là-bas" », Poésie et liturgie, $X I X^{e}-X X^{e}$ siècles, op. cit., p. 137-152. 
lire les lettres qui lui ont été écrites par de très nombreux ecclésiastiques ${ }^{40}$ pour comprendre à quoi il peut être fait allusion.

Claudel considère poètes et artistes comme des «Scribae, c'està-dire producteurs de l'expression »-selon le terme de l'Évangilequi sont « chercheurs diligents de la Perle unique, de la drachme perdue ou plutôt placée dans les profonds coffres-forts de l'Univers » et qui, par leurs moyens propres, recherchent « le Royaume des Cieux ${ }^{41} »$. C'est bien la leçon que professe encore le poète en 1937 dans Du sens figuré de l'écriture: "Nous sommes associés à une liturgie ${ }^{42}$ » référant implicitement à une autre idée qui lui est chère et qu'il explicite dans un texte de 1944, à savoir que le terme liturgie ne doit pas être pris dans un sens trop étroit : « Tout dans la Création est perpétration d'un Office [...]. L'Office liturgique proprement dit, celui dont le rôle de l'Église est de s'acquitter, ne fait qu'apporter Dieu $[\ldots]^{43}$ » Ainsi, la beauté du rite telle que la justifie Claudel participe d'une pensée religieuse totalisante : loin de s'en tenir à une domination des sens comme cela semble en partie le cas pour le personnage de Durtal chez Huysmans, elle est l'élément d'un tout, moment d' « une multiple représentation, au sens pictural et théâtral du mot» «de l'énorme Parabole ${ }^{44} »$. Elle n'est pas ornement, mais elle-même sacrée et sacralisante, à la fois issue du Créateur et réponse (ou répons) au Créateur.

Université de Cergy-Pontoise

UMR CNRS 7187 LDI

95000 Cergy-Pontoise

catherine.mayaux@u-cergy.fr

40. Voir Correspondance de Claudel avec les ecclésiastiques de son temps, Dominique Millet-Gérard éd., Paris, Honoré Champion, vol. I, 2005, vol. II, 2008.

41. « Note sur l'art chrétien», loc. cit., p. 131.

42. Du sens figuré de l'écriture, Le Poëte et la Bible, I, op. cit., p. 845.

43. Voir sur ce point La liturgie, l'église et la sainte Vierge, dans Le Poëte et la Bible, t. II, Paris, Gallimard, 2004, p. 308.

44. Voir « Note sur l'art chrétien », op. cit., p. 122-123 : «Autour de l'autel ce n'est plus une présence constitutionnelle, mais vraiment une assistance, une présence en acte, vivante et diverse, une multiple représentation, au sens pictural et théâtral du mot, dans le détail touffu de ses scènes entremêlées, du drame de notre Salut. La comparution est devenue un Office et pour l'alimenter tout le répertoire de notre vie actuelle, de la nature et des saisons, de la moralité et de l'histoire, des spectacles et des idées, a profité, en une espèce de sainte émeute qui se saisit des parois, qui escalade les clochers, qui s'interpose à toutes les coupées de la lumière, des dispositions et des niveaux de l'énorme Parabole ». 